\title{
Rechtsformen letztwilliger Verfügungen in den Wiener Stadtbüchern (1395-1430) Eine Bilanz aufgrund der vorliegenden Edition*
}

Das Archiv der Stadt und des Landes Wien verwahrt unter der Handschriftensignatur A 285/1-3 drei in Leder gebundene Foliobände, die üblicherweise als „Wiener Testamentsbücher" bezeichnet werden. Die Quelle nennt sich selbst "Stadtbuch" und ist ihrem Charakter nach auch diesem Rechtsquellentypus ${ }^{1}$ zuzuordnen.

\footnotetext{
*BRAUneder, JARItZ, Stadtbücher 1; BRAUnEDER, JARITZ, NESCHWARA, Stadtbücher 2; JARITZ, NESCHWARA, Stadtbücher 3; JARITZ, NESCHWARA, Stadtbücher 4. - Von den drei im Wiener Stadt- und Landesarchiv verwahrten Foliobänden des Stadtbuches im Umfang von 193, 293 und 373 Blättern konnte durch die bisher vorliegende Edition bereits mehr als die Hälfte im Druck veröffentlicht werden. Teil 4 schloss 2009 mit Blatt 245 des zweiten Foliobandes ab; für die Edition der übrigen Eintragungen aus Folioband 2 sowie jener aus Folioband 3 werden drei weiterer Teilbände in Aussicht genommen; der letzte Teilband wird ein Gesamtregister enthalten. Der Zugang zum edierten Material ist bis dato noch immer etwas schwierig, nämlich insofern, als umfassende Register erst nach dem Abschluss der Edition vorliegen werden, was bis 2015 verwirklicht sein soll. Die nun vorliegenden Bände enthalten bloß ein rechtshistorisches Glossar und ein kurzregestenartiges Verzeichnis der Eintragungen. Um dieses Problem zu mindern und als Vorbereitung für die Gesamtregister nach Abschluss der Edition werden im Internet [http://www. imareal.oeaw.ac.at/] (abgerufen am 31. 5. 2010) einige Register angeboten, welche die Benutzung der vorliegenden Bände erleichtern sollen. Diese sind als "work in progress" $\mathrm{zu}$ verstehen und repräsentieren den jeweiligen Stand ihrer Anlage; "updates“ erfolgen regelmäßig. Die Verweise in den Registern beziehen sich jeweils auf die vergebenen Nummern der Einträ-
}

Die Bezeichnung „Testamentsbuch" ist nicht zeitgenössisch, sie wurde von Friedrich Walter im Titel seiner 1923 am Institut für Österreichische Geschichtsforschung eingereichten Prüfungsarbeit „Die sogenannten Wiener Testamentsbücher (1395-1430) (Eine diplomatische Untersuchung) ${ }^{\star 2}$ verwendet, welcher die Bezeichnung der Quelle durch das Wiener Stadtund Landesarchiv als „Testament- und Geschäfts-Bücher" folgt. ${ }^{3}$ Die Verengung der Qualifikation des Inhalts durch diese Bezeichnungen, nämlich auf „Testamente“ bzw. auf „Testamente und Geschäfte", ist unzutreffend, und zwar in zweifacher Weise: Zum einen enthält das Stadtbuch nicht nur Eintragungen über letztwillige Verfügungen, und es sind diese auch keine „Testamente" ${ }^{\prime 4}$ im eigentlichen Sinn,

ge in der Edition (Teil 1 enthält Nr. 1-624; Teil 2 Nr. 625-1239, Teil 3 Nr. 1240-1866, Teil 4 Nr. 18672521). Weitere Register zu Orten, Personen und Sachen werden vorbereitet.

${ }^{1}$ Dazu KÖBLER, Zielwörterbuch 899; KLÖTZER, Stadtbuch, mit ausgiebigen Literaturangaben; vgl. künftig auch NESCHWARA, Rezensionen (im Erscheinen).

2 BRAUNEDER, JARITZ, Stadtbücher 1, 9f. - Walter selbst räumt freilich ein (Testamentsbücher 5, 15), dass die Bezeichnung der Stadtbücher als „Testamentsbücher" nicht zutreffend ist, allerdings unter dem quantitativen Aspekt, dass im Stadtbuch nur etwa die Hälfte der Eintragungen „Testamente“ betrifft.

${ }^{3}$ MAYER, Handschriften 33.

${ }^{4}$ In mehr als 2500 Eintragungen finden sich in der bisher vorliegenden Edition nur drei Belege für die Verwendung des Terminus „Testament“, und zwar 
sondern letztwillige Verfügungen eigener Art, nämlich zeitgenössisch sogenannte "Geschäfte". Zum anderen enthalten die Stadtbücher aber nicht nur (letztwillige Rechts-)Geschäfte, sondern auch zahlreiche weitere Eintragungen über Rechtsgeschäfte des Erb- und Familienrechts. Sie beschränken sich auch nicht auf die Evidenz von Rechtsakten privatrechtlicher Natur, sondern enthalten auch solche aus dem Bereich des öffentlichen Rechts. ${ }^{5}$

\section{Testament und Geschäft}

\section{A. Grundsätzliches ${ }^{6}$}

Der im Generalthema der Tagung verwendete Begriff Testament wurde im Titel des vorliegenden Beitrags daher bewusst vermieden, weil die in den Wiener Stadtbüchern eingetragenen letztwilligen Verfügungen keine Testamente im technischen Sinn darstellen.

Das Testament ist eine Entwicklung des römischen Rechts. ${ }^{7}$ Testamente orientieren die Rechtsnachfolge am Gesamtnachlass eines Erblassers, welcher durch Rechtsgeschäft eine (oder mehrere) Personen zur General- und Universalsukzession beruft. Geschäfte ${ }^{8}$ hingegen dienen im technischen Sinn der Zuordnung von einzelnen Gegenständen des Nachlasses oder

zweimal im Kontext einer inserierten lateinischsprachigen Urkunde in BRAUNEDER, JARITZ, Stadtbücher 1, Nr. 458 (hier testamentum als Synonym für Seelgerätstiftung) und Nr.31 (testamentis execucione als Synonym für Willensvollstreckung), sowie einmal im Index zu BRAUNEDER, JARITZ, NeSCHWARA, Stadtbücher 2, Nr. 910 (testamentum als Synonym für Geschäft).

${ }^{5}$ Siehe unten bei Anm. 60.

${ }^{6}$ Zum Folgenden grundsätzlich: LENTZE, Testamentsrecht. Weitere Literaturangaben PAUSER, Seuchentestament, 481f., besonders Anm. 15.

${ }^{7}$ OGRIS, Testament, mit weiteren Literaturangaben.

${ }^{8}$ BRAUNEDER, JARITZ, Stadtbücher 1, $17 \mathrm{f}$. von Nachlassmassen, ${ }^{9}$ welche nicht Kraft Gewohnheitsrechts oder aufgrund von anderen (sachen- oder ehegüterrechtlichen) Rechtsgeschäften ${ }^{10}$ bestimmten Rechtsnachfolgern bereits vorbehalten sind: Geschäfte ändern daher die gewohnheitsrechtliche Erbfolge ${ }^{11}$ nicht grundsätzlich $\mathrm{ab}$, sondern modifizieren sie bloß, und zwar insbesondere dadurch, dass sie den Kreis der Blutsverwandten als den vom Gewohnheitsrecht bestimmten Erben durch andere Personen, vor allem um den überlebenden Ehegatten, und um Institutionen, insbesondere die Kirche, erweitern; daher ist im heimischen Rechtsleben anders als nach römischem Recht ("nemo pro parte testatus, pro parte intestatus decedere potest" D 50, 17, 7) - auch eine Kumulation von Berufungsgründen - Geschäft neben Gewohnheitsrecht - im Erbrecht möglich. ${ }^{12}$

\section{B. Vergabungen von Todes wegen ${ }^{13}$}

Ursprünglich war das gewohnheitsrechtliche Erbrecht ausschließlich von sachen- und familienrechtlichen Instituten bestimmt: Das dem Erblasser zugeordnete Vermögen war seiner (letztwilligen) Disposition entzogen, weil es den

\footnotetext{
${ }^{9} \mathrm{Zu}$ den einzelnen Vermächtnis-Arten: LENTZE, Testamentsrecht 2, 184ff. (Sachgesamtheiten wie Hausgeräte; Geldvermächtnisse über bestimmte Summen, allenfalls als Alternativvermächtnis mit Wahlmöglichkeit des Bedachten; Forderungsvermächtnis; Schuldbefreiungsvermächtnis; Schuldvermächtnis; Rentenvermächtnis; Versorgungsvermächtnis; zur Abgrenzung von Anordnungen über die Liquidierung von (Erblasser-)Verbindlichkeiten sowie über die Erfüllung von offenen Rechtsgeschäften des Erblassers: ebd. 190ff.; zu Anordnungen über Begräbnis und Totenfeiern DERS., Begräbnis und Jahrtag; zu Messstiftungen DERS., Altarpfründe; Vgl. auch LUTZ, Alltagskultur und Lebensverhältnisse); DERNDARSKY, Analyse von Testamenten.

10 BRAUnEDER, Typen 331f.; WERKMÜLLER, Rechtsgeschäfte auf den Todesfall.

${ }^{11}$ BRAUNEDER, Typen 336f.

12 BRAUNEDER, Typen 338.

${ }^{13}$ Zum Folgenden grundsätzlich LENTZE, Testamentsrecht $1,100 \mathrm{ff}$.
} 
nächsten Blutsverwandten als Erben vorbehalten war; die Quellen ${ }^{14}$ sprechen von der "Wartung ", ${ }^{15}$ worin eine anwartschaftliche Position der nächsten Blutsverwandten auf künftigen Erwerb des Familienvermögens als „Erbgut “16 zum Ausdruck kommt. ${ }^{17}$ Ausgenommen von dieser Bindung des Familienvermögens ist von Seitenverwandten erworbenes "Erbgut" und vor allem sogenanntes "gewonnenes" Gut oder "Kaufgut", ${ }^{18}$ das der Erblasser selbst erwirtschaftet hat - allein oder gemeinsam mit anderen, vor allem dem Ehegatten. Erbgut, das von den Vorfahren herrührt, ist - nach dem Stand des Wiener Gewohnheitsrechts Mitte des 14. Jahrhunderts (Wiener Stadtrechtsbuch 134019) einer wirksamen Verfügung grundsätzlich entzogen, außer bei Zustimmung der warteberechtigten Erben durch „Erbenlaub“ ${ }^{\prime 20}$ oder bei erwiesenem Vorliegen von sogenannter "echter Not $^{\prime \prime 21}$ (unverschuldetem Notstand).

Eine Ausnahme von der Bindung des Familienvermögens an die warteberechtigten Erben bildete schon im Frühmittelalter der sogenannte „Freiteil“, ${ }^{22}$ wodurch dem Erblasser eine Quote des Familienvermögens für Verfügungen zugunsten der Kirche offenstand. Solche Vergabungen sind aber nicht als - einseitige - letztwillige Verfügungen konstruiert, sondern als -

${ }^{14}$ Dazu BRAUNEDER, Übertragung Wiener Rechts.

${ }^{15}$ OGRIS, Erbenwartrecht; DERS., NESCHWARA, Erbenlaub.

${ }^{16}$ OGRIS, Erbgut; aus romanistischer Sicht: WESENER, Ephemere Besonderheiten 1405f.

${ }_{17}$ Zum Anfechtungsrecht bzw. zur Abfindung übergangener gewohnheitsrechtlicher Erben: LENTZE, Testamentsrecht 1, 116 f.

${ }^{18}$ OGRIS, Kaufgut; OGRIS, NeschWARA, Erbenlaub.

${ }^{19}$ BRAUNEDER, Geltung obrigkeitlichen Privatrechts.

${ }^{20}$ OGRIS, Erbenlaub, 958f.; OGRIS, NeschWARA, Erbenlaub; zur Funktion des Geschäfts im Rahmen der Erbenauseinandersetzung: LENTZE, Testamentsrecht 1, $112 \mathrm{ff}$.

${ }^{21} \mathrm{Zu}$ Echter Not BRAUNEDER, JARITZ, Stadtbücher 1, 14f. Zum Konsens von Ehegattin und Erben: LENTZE, Testamentsrecht 1, $116 f$.

${ }^{22}$ OGRIS, Freiteil; DERS., Freiteil. zweiseitige - Schenkungen auf den Todesfall. ${ }^{23}$ Sie kommen also durch ein Rechtsgeschäft unter Lebenden zustande und binden daher den Erblasser schon zu Lebzeiten und der Begünstigte wird sofort Eigentümer, freilich bloß aufschiebend bedingt ${ }^{24}$ - und unter Umständen auch unter Vorbehalt des Fruchtgenusses. ${ }^{25}$ Sie stellen Stiftungen zur Sicherung des Seelenheils ${ }^{26}$ des Erblassers dar und haben konkrete Leistungen zugunsten geistlicher Einrichtungen oder Personen zum Gegenstand. ${ }^{27}$ Vergabungen von Todes wegen lassen als zweiseitig bindende Rechtsgeschäfte grundsätzlich auch keine Widerrufsmöglichkeit $\mathrm{zu}$, außer bei Vereinbarung des Verfalls bei Nichterfüllung des Stiftungszwecks oder eines Ablösevorbehalts zugunsten der Erben. ${ }^{28}$ Sie wirken später fort in sogenannten Gemächten, welche als sachenrechtliche Rechtsgeschäfte mit aufschiebender Wirkung zugunsten des überlebenden Ehegatten konstruiert sind und daher einer Schenkung auf den Todesfall entsprechen. Sie haben meist ehegüterrechtliche Funktionen. Ihre Weiterentwicklung durch die Vereinbarung von gegenseitigen Gemächten (, auf Überleben“29) führt schließlich zum Erbvertrag. ${ }^{30}$

\footnotetext{
23 OGRIS, Schenkung 1382f.

${ }^{24}$ Dazu LeNTZE, Testamentsrecht 2, $188 \mathrm{ff}$.

${ }^{25}$ DERS., Testamentsrecht 1, 105.

${ }^{26}$ Ebd. 148 sowie die oben in Anm. 9 angeführte Literatur.

${ }^{27}$ LENTZE, Seelgerät; dazu auch DERS., Altarpfründe.

${ }^{28}$ LENTZE, Testamentsrecht 1, 106. Zur Kürzung von Vermächtnissen: DERS., Testamentsrecht 2, $192 \mathrm{f}$.

${ }^{29} \mathrm{Zu}$ Miteigentum BRAUNEDER, JARITZ, Stadtbücher 1, $20 f$.

${ }^{30} \mathrm{Zu}$ Gemächt BRAUNEDER, JARITZ, NESCHWARA, Stadtbücher 2, 13.
} 


\section{Geschäfte ${ }^{31}$}

Geschäfte als einseitig konstruierte - und damit jederzeit widerrufbare ${ }^{32}$ - Rechtsgeschäfte kommen in Wien und im umliegenden Erzherzogtum Österreich im 13. Jahrhundert auf, sie sind zu Beginn des 14. Jahrhunderts vor allem im städtischen Rechtskreis bereits stark verbreitet und erreichen in der zweiten Hälfte des 14. Jahrhunderts auch den ländlichen Raum;33 zu Beginn des 15. Jahrhunderts hat sich die Rechtsform des Geschäfts im Rechtsleben durchgesetzt. Die „Testier"-Fähigkeit war - unabhängig von körperlicher Gesundheit, auch wenn sich in Geschäften fallweise Hinweise ${ }^{34}$ dazu finden -

${ }^{31}$ Zum Folgenden grundsätzlich LENTZE, Testamentsrecht. Vgl. dazu auch LoOSE, Hamburger Testamente IXff.; Paul BAUR, Testament und Bürgerschaft 11ff.; REINHARDT, Lüneburger Testamente VIIff.; MARQUARDT, Görlitzer Bürgertestamente 14ff. (Entwicklung der Testamente bis zum 16. Jahrhundert).

${ }^{32}$ Dennoch finden sich in Geschäften vereinzelt Hinweise auf das Recht zum Widerruf: LeNTZE, Testamentsrecht 1, 114ff.; DERS., Testamentsrecht 2, 193ff. Entsprechende Formeln lauten in der Regel, dass das Geschäft, solange es nicht widerrufen wird, „stet und unzebrochen" bleibe: siehe dazu auch

[http://www.imareal.oeaw.ac.at/] $\rightarrow$ Rechtshistorisches Sachverzeichnis: aufrecht [und unangefochten] bleiben, halten, sein (,sta[e]t [aufrecht]; sta[e]t [aufrecht] und unczebrochen"); vgl. BRAUNEDER, JARITZ, Stadtbücher 1, Nr. 70, 105, 126, 129, 216, 275, 286, 301, 303, 334, 341, 415, 425, 561, 592, 629; BRAUNEDER, JARITZ, NESCHWARA, Stadtbücher 2, Nr. 648, 755, 760, 788, 800, 801, 802, 865 („,staet und beleyblich“), 873, 877 (,,staet und unuerrukcht"), 896, 948, 950, 951, 960, 961, 963, 1013, 1023, 1024, 1071, 1116, 1187, 1205, 1209.

33 LENTZE, Testamentsrecht 1, 107ff.

${ }^{34}$ Ebda. 149f.; [http://www.imareal.oeaw.ac.at/] $\rightarrow$ Rechtshistorisches Sachverzeichnis: geschäftsfähig sein; vgl. BRAUNEDER, JARITZ, Stadtbücher 1, Nr. 301 (,mit gutem willen, mit wolbedachtem muet und mit gesuntem leib und mit gutem wizzen und sinnen"), Nr. 462 („,mit gutem willen und wohlbedachtem muet; mit guten wissen und sinnen und gesunden leib“), Nr. 561 (,,mit gutem wissen und sinnen und gesundem leib"), Nr. 592 (,,mit gutem willen und wohlbedachtem muet und gesundem leib"); BRAUNEDER, JARITZ, NESCHWARA, Stadtbücher 2, Nr. 661 („,mit gesuntem leyb“), Nr. 801 (,,mit bloß an das Erreichen der Volljährigkeit ${ }^{35}$ geknüpft, ${ }^{36}$ nach Wiener Stadtrecht also ab dem 18. Lebensjahr gegeben, und zwar unabhängig vom Geschlecht. Aufgrund der zahlreichen, für Wien von 1395 bis 1430 in den Stadtbüchern eingetragenen Geschäfte äußerte Lentze die Vermutung, ${ }^{37}$ dass nahezu jeder „Testier“-Fähige, vor allem die Erbbürger, eine letztwillige Verfügung getroffen haben könnte. Bei den höheren städtischen Schichten (Erbbürger, städtische Funktionäre, Beamte, Universitätsangehörige, Geistliche) überwiegt - auch zufolge ihrer Siegelfähigkeit $^{38}$ - die schriftliche Form, bei der Masse der unteren städtischen Schichten dominieren mündliche Verfügungen. ${ }^{39}$

Bei Fehlen von letztwilligen Verfügungen erfolgt die Feststellung der gewohnheitsrechtlichen Erben oft aufgrund von Verwandtschaftsweisungen; ${ }^{40}$ sie dienen - solange obrigkeitliche Personenstandsverzeichnisse fehlen - als Surrogat für Geburtsbescheinigungen.

guetem willen und mit wolbedachtem muet und mit gesundem leyb und mit gutem wiczen und sinnen"), Nr. 802 (,,mit wolbedachtem mut und mit gesundem leyb), Nr. 890 (,mit gutem wisczen und wolbedachtem mut und mit gesundem leyb“), Nr. 951 („,mit gutem wisczen, mit wolbedachtem mut und mit gesundem leib und mit gutem wiczen und synnen“), Nr. 1187 (,mit gutem willen und wolbedachtem muet und mit gutem wiczen und sinnen und mit gesundem leib“).

${ }^{35}$ LENTZE, Testamentsrecht 1, 149f.; zur Volljährigkeitsweisung JARITZ, NESCHWARA, Stadtbücher 4, 11f.

${ }^{36}$ Hinweise auf die Testierfreiheit im Sinne der Freiwilligkeit der getroffenen Verfügungen: vgl. Anm. 34.

37 LENTZE, Testamentsrecht 1, 116.

${ }^{38}$ HOLZHAUER, Unterschrift.

${ }^{39}$ [http://www.imareal.oeaw.ac.at/] $\rightarrow$ Verzeichnis der Eintragungen, Geschäft.

${ }^{40} \mathrm{Zu}$ Verwandtschaftsweisungen BRAUNEDER, JARITZ, Stadtbücher 1, 21. 


\section{Letztwillige Verfügungen im spätmittelalterlichen Wien}

\section{A. Allgemein}

Grundsätzlich lassen sich die im Spätmittelalter in Wien verbreiteten letztwilligen Verfügungen der Form der Beurkundung nach in öffentliche und private unterscheiden. $\mathrm{Zu}$ den privaten zählen vor allem die Siegelurkunden;41 sie sind in subjektiver Fassung konzipiert, ursprünglich in Latein, seit dem ersten Viertel des 14. Jahrhunderts aber grundsätzlich in deutsch verfasst. Sie lassen sich weiters in fremdhändig und eigenhändig geschriebene unterscheiden, eigenhändig auch unterschriebene Siegelurkunden liegen nur selten vor. Sie sind mit eigenem Siegel (oder Petschaft) versehen und ermöglichen daher eine Beurkundung in eigener Sache, oft werden fremde Siegel (oder Petschaft) von Zeugen zur Beglaubigung verwendet. ${ }^{42}$ Im Spätmittelalter entwickelt sich ein Formular für den formalen Aufbau der Urkunden mit Hinweisen auf das Erfordernis der öffentlichen Kundmachung, fallweise auf die vorhandene Geschäftsfähigkeit des Erblassers und die Rechtmäßigkeit seiner Anordnungen, selten auch auf den Konsens der Erben. ${ }^{43}$ Von der Disposition (letztwillige Verfügung) abgesehen enthält es Angaben über die beigezogenen Zeugen, die Art der Besiegelung und das Datum der Anordnungen.

Die öffentliche Beurkundung beruht auf der Delegierung obrigkeitlicher Gewalt zur Errichtung einer letztwilligen Verfügung vor einem Notar oder Offizialat im städtischen bzw. Pfarrer im ländlichen Raum (kanonisches Testament), oder es erfolgt eine unmittelbare Beurkundung durch den Rat. ${ }^{44}$ Die Beurkundung geschieht in objektiver Fassung, die Sprache der

\footnotetext{
${ }^{41}$ LENTZE, Testamentsrecht 1, $118 \mathrm{ff}$.

${ }^{42}$ Ebd. $120 f$.

${ }^{43}$ Ebd. 116f.

${ }^{44}$ LENTZE, Rat und Wiener Testamentsrecht.
}

Urkunden ist in Einzelfällen Latein; der Aufbau des Urkundenformulars ist stark formalisiert. Öffentliche Beurkundungen sind im Rechtsleben selten zu konstatieren, Notariatsinstrumente und Offizialatsurkunden sind vereinzelt überliefert; auch die Beurkundung durch den Rat ist nur sporadisch $\mathrm{zu}$ belegen. ${ }^{45}$ Sie bildet den Übergang zum Bucheintrag als Beurkundungsmittel für letztwillige Verfügungen. Die dafür von 1395 bis 1430 verwendeten Stadtbücher sind nicht die ältesten Quellen der für Wien überlieferten Rechtsgeschäfte. ${ }^{46}$

\section{B. In den Stadtbüchern}

\section{1. Über Wiener Stadtbücher im Allgemeinen}

Der Beginn der Existenz von Stadtbüchern in Wien ${ }^{47}$ ist nicht genau bestimmbar. Für die Zeit um 1320 gibt es Hinweise auf die Existenz eines allgemeinen Stadtbuches, das "große" Stadtbuch, später - nach den Beschlägen auf den Buchdeckeln - auch „Eisen“-Buch genannt. ${ }^{48}$ Es dient der Evidenz der Quellen des Stadtrechts als Grundlagen der Stadt-,,Verfassung“.49 Seit Mitte des 14. Jahrhunderts liegen auch Hinweise auf Stadtbücher für privatrechtliche Eintragungen, sogenannte Rechtsgeschäftsbücher, vor, welche hauptsächlich als Grundbücher fungieren. ${ }^{50}$ Es lassen sich verschiedene Typen differenzieren: Für 1368 bis 1419 ist ein sogenanntes Kaufbuch überliefert, das 1420 im sogenannten Gewerebuch aufgeht, welches seinerseits für 1373 bis 1517 überliefert ist. Für etwa denselben Zeitraum, von 1373 bis 1419, liegt ein sogenann-

\footnotetext{
${ }^{45}$ DERS., Testamentsrecht 1, $124 \mathrm{ff}$.

${ }^{46}$ Das Stadtbuch enthält dafür einen einzigen Beleg aus dem Jahr 1396: BRAUNEDER, JARITZ, Stadtbücher 1, Nr. 89a.

${ }^{47}$ LENTZE, Testamentsrecht (1952) $126 \mathrm{f}$.

${ }^{48}$ Demelius, Wiener Eisenbuch 47ff.; OpLl, Großes Wiener Stadtbuch 7ff.

${ }^{49}$ CSENDES, Rechtsquellen $19 \mathrm{f}$.

${ }^{50}$ Zur historischen Entwicklung der Grundbücher in Wien: LOHRMANN, Grundbücher 1ff.
} 
tes Verbotsbuch vor, welches 1420 im sogenannten Satzbuch aufgeht, das wiederum für 1373 bis 1523 überliefert ist. ${ }^{51}$ Seit Beginn der Neuzeit bestehen als Grundbücher ${ }^{52}$ bezeichnete Verzeichnisse für die Evidenz der Rechtsverhältnisse an den städtischen Liegenschaften.

\section{2. Über die Stadtbücher von 1395-1430 im Besonderen}

a) Wesen

Das für Ende 1395 bis 1430 in drei Bänden vorliegende Stadtbuch dient dagegen der Evidenz von Rechtsverhältnissen familien- und erbrechtlicher Natur; 53 es wurde daher - wie eingangs erwähnt - auch irrig als „Testamenten“-Buch ${ }^{54}$ bezeichnet, obwohl es auch damit in Verbindung stehende zahlreiche andere Typen von Rechtsgeschäften enthält. ${ }^{55}$ Sein rechtspolitischer Zweck liegt - so wie bei allen Rechtsgeschäftsbüchern - in der Sicherung der Publizität von Rechtszuständen; es entfaltet in gewisser Weise öffentlichen Glauben. Die - unabhängig von Urkunden - bewirkten Eintragungen sind für die betreffenden privatrechtlichen Rechtsverhältnisse konstitutiv. ${ }^{56}$

Die Sprache der Eintragungen ist nahezu ausschließlich Deutsch; es sind bloß einige lateini-

\footnotetext{
${ }^{51}$ Von den mittelalterlichen Grundbüchern der Stadt Wien ist ein Teil (Kaufbuch A 1368-1372 und C 13731388; Satzbuch A1 1373-1388; Gewerebuch B 13731419; Verbotsbuch 1373-1399) im Druck veröffentlicht worden: STAUB, Älteste Kaufbücher; STAUB, Gewerbuch B. Verbotbuch; STAUB, Satzbuch A; zu den anderen im Wiener Stadt- und Landesarchiv verwahrten Rechtsgeschäftsbüchern: LOHRMANN, Grundbücher 7.

52 LOHRMANN, Grundbücher; vgl. allgemein zur Institution von Grundbüchern: NESCHWARA, Grundbuch.

${ }^{53}$ Vgl. zu anderen „Testamentenbüchern“ beispielsweise: MAJOROSSY, SZENDE, Preßburger Protocollum Testamentorum.

${ }^{54}$ Siehe oben bei Anm. 2ff.

${ }^{55}$ LENTZE, Testamentsrecht 1, $126 \mathrm{ff}$.

${ }^{56}$ Ebd. 133f., 137.
}

sche Urkunden-Abschriften $\mathrm{zu}$ konstatieren. ${ }^{57}$ Der Eintragungsmodus ist nicht einheitlich; den Regelfall (mehr als 90 \%) bildet das mündliche Vorbringen mit anschließender Protokollierung und Eintragung im Stadtbuch, gefolgt von Mitteilungen über vor auswärtigen Obrigkeiten getätigte Rechtsakte (7,5\%; fast alle betreffen Verwandtschaftsweisungen) sowie von Entscheidungen über Rechtsstreitigkeiten im Rat (1,5\% der Eintragungen); dagegen ist von einer Ausnahme abgesehen kein einziger Fall über die Errichtung eines Rechtsgeschäfts vor dem Rat zu konstatieren. ${ }^{58}$

\section{b) Die Eintragungen}

Die im Stadtbuch enthaltenen Eintragungen betreffen etwa zur Hälfte Geschäfte, mehr als ein Drittel aller Eintragungen enthält die Bestellung von Vormündern (7\%) und/oder die Bestellung von Willensvollstreckern (29\%); den zweiten Schwerpunkt bilden Verwandtschaftsweisungen (30\%); die übrigen privatrechtlich relevanten Eintragungen verteilen sich auf Volljährigkeitsweisungen (4\%) und Erbrechts-Urteile (1,5\%); der Rest (14,5\%) betrifft Rechtsakte des öffentlichen Rechts; sie verteilen sich auf (mehrere) Handwerksordnungen, stadtherrliche Verfügungen und Verbote (hauptsächlich zum Handwerks- und Abgabenwesen), einige betreffen Kundmachungen des Landesfürsten und Herrschaftsverträge der Dynastie; hinzu kommen Quellen der „Stadtverfassung“ wie Ratslisten (Mitglieder des äußeren und inneren Rats), vereinzelt Belege über Bürgereide oder den Amtsantritt des Stadtschreibers, Ergebnisse von Bürgermeisterwahlen, Petitionen und Treuegelöbnisse der Bürgergemeinde oder die Bestel-

\footnotetext{
${ }^{57}$ Ebda. 128; Belege in den vorliegenden Editionen: BRAUNEDER, JARITZ, Stadtbücher 1, Nr. 31; JARITZ, NESCHWARA, Stadtbücher 3, Nr. 1557.

58 Siehe oben Anm. 45.
} 
lung eines städtischen Aufgebots auf Anordnung des Landesfürsten. ${ }^{59}$

\section{Die Geschäfte}

\section{a) Eintragungsmodus}

Bei den Eintragungen über Geschäfte haben sich grundsätzlich drei Typen von Formularen herauskristallisiert: Vorbringen des Geschäfts durch Verwandte und Zeugen vor dem Rat (Typ A) oder durch Zeugen allein (Typ B); in beiden Fällen findet sich die publizitätswirksame eidesstattliche Erklärung der Zeugen am Schluss der Eintragung. Davon ist eine Variante zu unterscheiden (Typ C), bei der sich die eidesstattliche Erklärung sogleich am Beginn der Eintragung findet: Typ A ist von 1395 bis 1411 und später wieder ab 1428 häufiger festzustellen; Typ B häufiger ab 1411 und wird danach nahezu gänzlich von Typ $C$ verdrängt. Mitteilungen von Geschäften durch auswärtige Obrigkeiten bilden seltene Ausnahmen (0,5\%). ${ }^{60}$

Die eidesstattliche Erklärung differiert je nach Amt oder Beruf der Zeugen; allgemein fungieren als Zeugen zumeist angesehene Ratsbürger ("Genannte“) oder andere „Biedermänner“, insbesondere auch Geistliche.61 Die Zahl der beigezogenen Zeugen variiert; sie wird mit stadtherrlicher Verordnung 1361 auf mindestens

\footnotetext{
${ }^{59} \mathrm{Vgl}$. die einschlägigen Stichwörter im Verzeichnis der Eintragungen [http://www.imareal.oeaw.ac.at/].

${ }^{60}$ LENTZE, Testamentsrecht (1952) 129-131, folgt der Typisierung von WALTER, Testamentenbücher 16ff.; vgl. dazu die Differenzierung der Typen durch BRAUNEDER, JARITZ, Stadtbücher 1, 17f., welcher auch die weiteren Editionen der Stadtbücher folgen.

${ }^{61}$ LeNTZE, Testamentsrecht (1952) 129ff.; zu den Genannten: WAHLE, Wiener Genannte 636-652. Nur einmal ist 1401 auch ein Jude (Michael Judenmayr [?]) als Zeuge zu konstatieren, BRAUNEDER, JARITZ, NESCHWARA, Stadtbücher 2, Nr. 643. Entsprechend differenziert ist auch die Diktion der Eidesformel: LENTZE, Testamentsrecht 1, 130; vgl. Rechtshistorisches Sachregister in [http://www.imareal.oeaw. ac.at/] Eidesstattliche Erklärung.
}

zwei festgelegt. Zeugen oder durch das Geschäft begünstigte Personen sind als Willensvollstrecker grundsätzlich ausgeschlossen und können nur aufgrund spezieller obrigkeitlicher Bestellung (nach Verzicht auf Rechte aus dem Geschäft) zugelassen werden. ${ }^{62}$

\section{b) Wege der Errichtung63}

Bei mündlicher Errichtung fungieren die beigezogenen Zeugen als Adressaten des letzten Willens und bringen die Anordnungen später im Rat vor; entweder unmittelbar nach erfolgter Verfügung mündlich oder unter Vorlage von schriftlichen Aufzeichnungen (,Zettel“). Im Rat erfolgt sodann entweder die Protokollierung des mündlichen Vorbringens und die (später) anschließende Eintragung der Verfügungen im Stadtbuch, oder es wird die vorgelegte Urkunde in Abschrift in das Stadtbuch inseriert. Dasselbe geschieht bei schriftlicher Verfügung durch den Erblasser, und zwar durch Vorlage einer eigenhändig ausgestellten ${ }^{64}$ oder fremdhändig verfassten Urkunde. Die Einbringer der Urkunden werden in der auf Anordnung des Rats im Stadtbuch vorgenommenen Eintragung oft nicht erwähnt, in Einzelfällen wird auf das Ersuchen des Erblassers zur Eintragung hingewiesen. Die Eintragung von schriftlichen Geschäften ist für die Wirksamkeit der darin enthaltenen Verfügungen des Erblassers aber nicht konstitutiv, ${ }^{65}$ sondern dient bloß dem Schutz vor Verlust der Urkunde. Die Eintragung solcher Urkunden erfolgt oft zu einem späteren Zeitpunkt als dem Ausstellungsdatum der Urkunde, oft Jahre nach

\footnotetext{
${ }^{62}$ LENTZE, Testamentsrecht 1, 131; JARITZ, NESCHWARA, Stadtbücher 3, 11f.

${ }^{63}$ LENTZE, Testamentsrecht 1, 131ff.

${ }^{64}$ Dazu eingehend ebd. 134ff., $137 \mathrm{ff}$.

${ }^{65}$ Ebd. 133f.; in der Regel wird auf die später vorzunehmende Eintragung im Stadtbuch hingewiesen: ebd. 137.
} 
Errichtung des Geschäfts. ${ }^{66}$ Unter den schriftlichen Geschäften ist eine große, verwirrende Vielfalt von Formen zu konstatieren. ${ }^{67}$

Der Anteil der mündlichen Geschäfte überwiegt deutlich den der schriftlichen. Es ergibt sich ein Verhältnis von etwa 3:1, bezogen auf den Beobachtungszeitraum zeigen sich in den vier vorliegenden Teilen der Edition leichte Schwankungen (Teil 1: 77:23\%; Teil 2: 72:28 \%; Teil 3: 73:27\%; Teil 4: 74: $26 \%$ ). ${ }^{68}$

c) Verbindung von Geschäften mit der Bestellung von Vormündern (Sachwaltern) und Willensvollstreckern ${ }^{69}$

Bei mehr als einem Drittel der im Stadtbuch verzeichneten Geschäfte ist eine Verbindung von letztwilliger Verfügung und Vormundschaft (Sachwalterschaft) (7\%) und/oder Willensvollstreckung (29\%) festzustellen, ${ }^{70}$ wobei tendenziell nicht nur allgemein eine quantitative Zunahme der Einsetzung von Willensvollstreckern zu beobachten ist, sondern vor allem auch eine Zunahme der obrigkeitlichen Bestellung. Die Aneignung des Nachlasses durch die gewohnheitsrechtlich oder rechtsgeschäftlich bestimmten Erben wird somit durch die Abwicklung des Nachlasses mittels privatrechtlich durch den Erblasser bestellter Willensvollstrecker abgelöst. Zunächst steht die Einsetzung von Willensvollstreckern oft auch in Verbindung mit Seelgerätstiftungen. Die Funktion des Willensvollstreckers entspricht einer Art Verwaltungs-

\footnotetext{
${ }^{66}$ Vgl. die Verzeichnisse der Urkunden, deren Datum von jenem der Eintragung abweicht, in JARITZ, NESCHWARA, Stadtbücher 3, 20, und Stadtbücher 4, 19.

${ }^{67}$ LENTZE, Testamentsrecht 1, $133 \mathrm{ff}$.

${ }^{68} \mathrm{Vgl}$. dazu die einschlägigen Stichwörter im Verzeichnis der Eintragungen in[http://www.imareal. oeaw.ac.at/].

${ }^{69}$ LENTZE, Testamentsrecht 1, 195ff.; vgl. zu Willensvollstreckern JARITZ, NESCHWARA, Stadtbücher 3, 11f.

${ }^{70} \mathrm{Vgl}$. dazu die einschlägigen Stichwörter im Verzeichnis der Eintragungen

[http://www.imareal.oeaw.ac.at/]
}

treuhand; seine eigentümergleiche Stellung belässt unter Umständen auch treuwidrige Verfügungen in Wirksamkeit.71 Die Besitzeinweisung in das Nachlassvermögen durch den Willensvollstrecker erfolgt durch sogenannte Einantwortung. ${ }^{72}$ Die vorliegende Edition spiegelt eine zunehmende Tendenz zur Einschaltung von Aufsicht und Kontrolle durch die städtische Obrigkeit im Nachlassverfahren wider; auch die zunächst nur fallweise anzutreffende Einbindung des Landesfürsten in das Nachlassverfahren, durch die Bestätigung von Geschäften oder die Einsetzung als oberster Willensvollstrecker, tritt im Laufe der Jahre häufiger hervor. ${ }^{73}$ Vor allem bahnt sich - außerhalb geistlicher Jurisdiktion und neben der Universitätsverwaltung mit der anwachsenden Häufigkeit der obrigkeitlichen Bestellung von Willensvollstreckern durch den Rat in Ansätzen auch im weltlichen Bereich ein gerichtliches Nachlassverfahren an.

\section{d) Mitteilungen von Geschäften/der Erbfolge}

Mitteilungen über die Errichtung von Geschäften durch auswärtige Obrigkeiten - abgesehen von sogenannten kanonischen Testamenten (sogleich 3.b) - an den Stadtrat von Wien sind selten; entsprechende Eintragungen darüber sind vereinzelt auch erst ab $1410 \mathrm{zu}$ konstatieren. ${ }^{74}$

\footnotetext{
${ }^{71}$ Zur Rechtsmacht und den Rechtspflichten der Willensvollstrecker: LENTZE, Testamentsrecht 2, $201 \mathrm{ff}$.

72 Zur Einantwortung BRAUNEDER, JARITZ, Stadtbücher 1, 15f.; vgl. aus romanistischer Sicht auch WESENER, Einantwortung.

${ }^{73}$ LENTZE, Testamentsrecht 1, 145; vgl. JARITZ, NESCHWARA, Stadtbücher 3, 11f.

${ }^{74}$ JARITZ, NesCHWARA, Stadtbücher 3, Nr. 1616 (Stadtgemeinde Ödenburg 1410), Nr. 1677 (Stadtgemeinde Wiener Neustadt 1410), Nr. 1713 (Herrschaft Lichtenstein 1410); JARITZ, NESCHWARA, Stadtbücher 4, Nr. 1888 (Stadtgemeinde Brünn 1412), Nr. 1998 (Stadtgemeinde Klosterneuburg 1413), Nr. 2082 (Stadtgemeinde Pressburg 1414), Nr. 2096 (Stadtgemeinde Baden 1414).
} 


\section{Sonderformen letztwilliger Verfügungen ${ }^{75}$}

a) Gemeinschaftliche Geschäfte -

Geschäft und Gemächt - Gemächte ${ }^{76}$

Neben dem - einseitigen - Geschäft als Haupttypus der im Stadtbuch enthaltenen letztwilligen Verfügung sind vereinzelt gemeinschaftliche Geschäfte unter Ehegatten zu konstatieren: Äußerst selten als Kombination von Verfügung des Ehemannes mit Konsens der Ehefrau; häufiger in Gestalt von Doppelgeschäften als bloß zeitgleiche Verfügungen von Ehegatten und vereinzelt als wechselseitige Verfügungen mit der Abrede auf Überleben als Erbvertrag zugunsten des Überlebenden. In spezifisch ehegüterrechtlicher Funktion begegnen in Einzelfällen auch mit Gemächten kombinierte Geschäft unter Ehegatten. ${ }^{77}$

\section{b) Notariell beglaubigte Geschäfte ${ }^{78}$ kanonische Testamente}

Seltene Ausnahmen - mit bloß zwei Belegen in den vorhandenen Editionen des Stadtbuches sind lateinische Notariatsinstrumente ${ }^{79}$ über letztwillige Verfügungen ${ }^{80}$ oder bloß notariell beglaubigte Urkunden über Geschäfte heimischen Rechts; ${ }^{81}$ zumeist handelt es sich um Verfügungen von Geistlichen; in einem Fall handelt es sich um einen Chorherrn zu St. Stephan, im anderen um einen Altarpriester zu St. Stephan sowie St. Michael in Wien.

\footnotetext{
75 LENTZE, Testamentsrecht 1, 141ff.

${ }^{76}$ Ebd. 146ff.; DERS., Testamentsrecht 2, $210 \mathrm{ff}$.

${ }_{77} \mathrm{Zu}$ Gemächten JARITZ, NeSCHWARA, Stadtbücher 3, $11 \mathrm{f}$.

${ }^{78}$ LENTZE, Testamentsrecht 1, 134; DERS., Testamentsrecht 2, 188 .

${ }^{79} \mathrm{Zu}$ Terminologie, theoretischen Grundlagen, Wesen und Formen der Notariatsurkunde: NESCHWARA, Notariat $19 \mathrm{ff}$.

${ }^{80}$ BRAUNEDER, JARITZ, Stadtbücher 1, Nr. 31.

${ }^{81}$ JARITZ, NESCHWARA, Stadtbücher 3, Nr. 1557; siehe unten Anhang D.
}

So wie notariell errichtete letztwillige Verfügungen begegnen im Stadtbuch ganz vereinzelt auch Eintragungen über Offizialatsurkunden und kanonische Testamente. Sie sind - wie die notariellen Beurkundungen ${ }^{82}$ - der weltlichen Sphäre des spätmittelalterlichen Rechtslebens von Wien nahezu fremd geblieben. ${ }^{83}$

\section{Anhang: Textbeispiele ${ }^{84}$}

\section{A. Mündliches Geschäft:}

Einzelne Verfügung zugunsten der Ehefrau ${ }^{85}$ [Stadtbuch II, fol. 165v] ,[...] [ ${ }^{a}$ Des nachsten sambstags vor sand Jorgen taga kom fuer den rat der stat zue Wienn frawe ${ }^{b}$ Annab, ${ }^{c}$ Hannsen seligen des Pek-

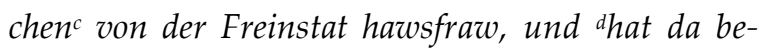
weist mit den erbern leuten, als si zue recht solt, mit Chonraten beim Goczakcher und mit Fridreichen dem Prantesserd, das gescheft, so der vorgenant Hanns an seinen lesten zeiten getan hat. Item eer hat geschaft der egenanten Annen, seiner hawsfrawn, sein haus gelegen in Sand Johannstrass zue Wienne zenechst Andres haus von Brunn, also fdas si sein geltschuld dauon ausrichten solf. Und sdas ubrig sol ir ledichleichen sein zue verseczen und zue verchauffens. Als darumb die obgenanten erbern leut,

\footnotetext{
${ }^{22} \mathrm{Vgl}$. NeSCHWARA, Notariat $45 \mathrm{ff}$.

83 LENTZE, Testamentsrecht 1, 134f., $143 \mathrm{ff}$.

${ }^{84} \mathrm{Im}$ Original übereinandergestellte Buchstaben werden im Folgenden - aus technischen Gründen - nebeneinanderstehend wiedergegeben.

${ }^{85}$ JARITZ, NESCHWARA, Stadtbücher 4, Nr. 2059: privat, mündlich, Typ A.

a-a 2. 4. 1414.

b-b Einbringerin: die Witwe des Erblassers.

c-c Der Erblasser.

d-d Zeugen der Verfügung.

e-e Verfügung zugunsten der Ehefrau.

f-f Verpflichtung der Ehefrau zur Schuldenerfüllung. g-g Der nach Schuldenabzug verbleibende Wert des Hauses steht der Witwe zur freien Verfügung.
} 
Chonrat beym Goczakcher hmit seinen trewn an aides stat und Fridreich Prantesser mit seinem starkchen aid habent gesagth, als si zue recht solten. $[\ldots]^{\prime \prime}$

\section{B. Mündliches Geschäft:}

Verfügungen zugunsten des Sohnes; Bestellung eines Vormundes; Bestellung der Einbringer zu Willensvollstreckern, obrigkeitlich ${ }^{86}$

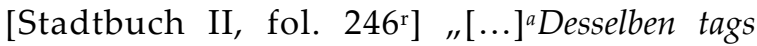
[= Samstag vor St. Bartholomäus] ${ }^{a}$ komen fuer den rat der stat ze Wienn die erbern lewt bHanns vom Kampp und Peter der Reaneyes ${ }^{b}$ und chabend da gesagt beye irn trewn an aides statc zue rechter zeit, als sye zurecht solten, umb das gescheafft, das fraw ¿Agnes die Verberinnd selige vor Kernertor getan hat. Von erst ehat sy geschaft irm sun Andren ier vier weingerten ${ }^{e}$, die ir vaeterlichs und muterleichs erb sind, ainer gelegen an der Hochenwart, des ain ganczes jeuch ist, zeneachst des Official weingarten, der ander gelegn in dem Gotschalichsperg, des zway drittail sind, zenechst der herren weingarten von den Schotten, item der dritt gelegen ze Praitensee in dem Altenperg, des ain halbes jeuch ist, zenêchst Otten des Hakcher weingarten. Item der vierd gelegen in dem Amaispach, des drew virtail sind, zenechst des von Starichenwerg weingarten ledichleich allen seinen frumen damit ze schaffen. Und fsol auch Andre, mein wiert, den egenanten Andren, meinen sun, den ich mit im hab, mit leib und mit guet inn-

h-h Eidesstattliche Bekräftigung der Richtigkeit der Verfügungen durch die Zeugen, ständisch differenziert.

${ }^{86}$ JARITZ, NESCHWARA, Stadtbücher 4, Nr. 2473; privat, mündlich, Typ C

a-a 21. 8. 1417.

b-b Einbringer als Zeugen.

${ }^{c-c}$ Eidesstattliche Bekräftigung der Richtigkeit der Verfügungen durch die Zeugen.

d-d Erblasserin.

e-e Verfügungen zugunsten des minderjährigen Sohnes.

${ }^{\mathrm{f}-\mathrm{f}}$ Bestellung des Vaters als Vormund für den minderjährigen Sohn. habent alslanng suencz das er zue seinen beschaiden jarn chuembts und sich selber verwesen mag. Item ${ }^{h}$ sye, hat geschaft, ob derselb ier suen mit dem tod abgieng ee denn er zue seinen beschaiden jarn keam und vogtpêr wuerd, so sol der egenant ier wiert die obgenanten vier weingaerten dennoch innhaben, nuczen und niessen unuerchumert uencz an seinen tod ${ }^{h}$. Und denn inach seinem tod so sullen denn die obgenanten vier weingaerten auf irn sun hern Hainreichen, pharrêr ze Chuelb, erben und geuallen ${ }^{i}$. Und jer sol dann darauf stiften ain ewige mezzi hincz Sand Cholman auf dem Gotsakchêr ze Wienn. Und ob das kwêr, das derselb her Hainreich, ir sun, abgieng mit dem tod ee dann die obgenant ewig mezz gestift wuerd, so sullen ir nachst frewnt die vorgenanten vier weingearten innemen und dann die egenant mezz darauf stiften ${ }^{k}$, als vor gemelt ist. Und 'das obgenant geschêfft hat sy ausczerichten enpholhen den egenanten Hannsen vom Champp und Petern dem Reaneyes, irn sweagearn. Und mwenn die benanten erbern lewt nach der statrecht darumb nicht gesagen mochten, seind es in enpholhen was, also habend es die herren des rats von in aus den hannden genomen und in das hinwider in ir trew enpholhen ${ }^{m}$ in der weise, als vor geschriben stet."

g-g Dauer der Vormundschaft bis zur Erreichung der Volljährigkeit (,zu seinen beschaiden jarn chumbt“).

h-h Bei Vortod des bedachten Sohnes vor Erreichung der Volljährigkeit Anordnung eines Leibgedinges.

${ }^{i-i}$ Bei Ableben des Ehemannes Anordnung einer Ersatzerbschaft zugunsten des älteren Sohnes.

j-j Verpflichtung des älteren Sohnes zur Stiftung einer ewigen Messe für die Erblasserin.

k-k Bei Vortod des älteren Sohnes Rückfall der Erbgüter an die nächsten Blutsverwandten der Erblasserin mit der Verpflichtung zur Anordnung der Messstiftung.

${ }^{1-1}$ Bestellung der Einbringer zu Willensvollstreckern. m-m Wegen Ausschluss der Einbringer von der Übernahme der Willensvollstreckung nach Stadtrecht Übertragung der Willensvollstreckung auf die Einbringer auf Anordnung des Stadtrates. 


\section{Schriftliches Geschäft:}

Verfügungen zugunsten von Ehegattin und nahen Familienangehörigen; Vormundschaftsbestellung für einen minderjährigen Bedachten; Verfügungen zugunsten kirchlicher (Seelgeräte) sowie sozialer Einrichtungen und zugunsten familienfremder Personen; Messstiftungen; Anordnungen über Schulden und Forderungen sowie zum Begräbnis; Bestellung eines Willensvollstreckers ${ }^{87}$

[Stadtbuch II, fol. 198v] „[...] aDes nachsten phincztags nach sand Agnesen tag der heyligen junckfrawen ${ }^{a}$ koemen fuer den rat der stat ze Wienn ${ }^{b}$ Hanns und Ulreich geprudear die Schaffswol und habent da beweist $t^{b}$ mit den erbern lewten zue rechtear zeit, als sy zurecht solten, cmit Meanndlein dem Hoepflein, dem vischear, und mit Heinreichen dem Scheppachc, das gescheafft, so ier brudear dThoman seligear der Schaffswold an seinen leczten zeiten getan hat, eals das an ainer zedel vor offem rat der stat ze Wienn gelesen und verhort ward und lawttet von wort ze wort, als hernach geschriben stete, Hie ist vermerkcht [fol. 199r] das gescheafft, daz ich Thoman der Schaffswol hinder mein lassen hab, als ich das geordent und geschafft hab fmit gueten wiczen und syennen und mit gueter vorbetrachtuengf $z$ u der zeit, da ich es wol getuen moecht, als hernach ausgenommen und begriffen ist. Item vonerst sschaff ich meiner hausfrawen Elzbeten mein haws gleich halbs gelegen vor Werdertoer ze Wienn zeneagst dem Ungruen. Und mit dem benanten halben haws sol und mag die vorgenant mein hausfraw allen iern frummen schaffen mit verchauffen, mit verseczen, wie und wenn sey des verlusst an all irrung und meanikleichs irrueng

${ }^{87}$ JARITZ, NESCHWARA, Stadtbücher 4, Nr. 2234: privat, schriftlich, Typ A (ähnlich B).

a-a 23. 1. 1416.

b-b Zwei Einbringer.

c-c Zwei Zeugen.

d-d Erblasser.

e-e Vorlage einer Urkunde.

f-f Hinweis auf die Testierfähigkeit.

g-g Verfügung zugunsten der Ehefrau (ein halbes Haus zur freien Verfügung). und hindernuesss. Item darnach ${ }^{h}$ schaff ich der benanten meiner hausfraw sechs phund phenning geltes, die ir die Dewtschenherren ze Wienn ier lebteag alle iar dauon rayhen und dienn süllen ${ }^{h}$ nach sag und lawt des briefs, den ich von in darumb gehabt hab und den die egenant mein hawsfraw innhat. Item darnach ischaff ich ier dreissig phund phenning auf ainem weingarten ${ }^{i}$ gelegen an dem Liechtenstain, der mir und meiner hausfrawn stet won dem Englisch. Item und ischaff ier auch das gelt, das mir der weinczuerl schuldig isti, der mir denselben weingarten von dem Englisch pawt, damit sye denselben weingarten dester pas pawn mag. Item so ${ }^{k}$ schaf ich ier aindlef phund phenning, die von meins ayden wegen herkoemen sind dacz den Dewtschenherren ${ }^{k}$. Item darnach 'schaf ich meins weibs bruder kynnd fuenfzehen phund phenningl und dasselb mgelt sol die obgenant mein hausfraw innhaben uncz daz dasselb kynnd zu seinen beschaiden jarn kumbtm. Item und ${ }^{n}$ schaff auch was dann des chlainen hausgeschier ist uber das vorgenant mein gescheafft, daz schaff ich der vorgenanten Elzbeten, meiner hausfrawen ${ }^{n}$. Item und oschaff auch meins brudear suen dem Nicklein ein dekchpet und ain ander pet und vier leylachen und zwaye kuess und ein ledearlachen ${ }^{\circ}$. Und ${ }^{p}$ schaff im auch ein jeuch weingarten ${ }^{p}$ gelegen dacz Sand Veyet und haisset der Chrannest. Also "ob das bescheach,

\footnotetext{
h-h Weitere Verfügung zugunsten der Ehefrau; Rentenbezugsrecht gegen den Deutschen Orden.

${ }^{\mathrm{i}-\mathrm{i}}$ Weitere Verfügung zugunsten der Frau (auf einem Weingarten sichergestellter Betrag). j-j Schuldenlegat zugunsten der Ehefrau.

k-k Weitere Schuldenabtretung zugunsten der Ehefrau.

${ }^{1-1}$ Geldlegat an einen minderjährigen Neffen der Ehefrau.

m-m Bis Erreichung der Volljährigkeit Vorbehalt des Nutzungsrechts zugunsten der Ehefrau.

${ }^{n-n}$ Verfügung des Hausrats zugunsten der Ehefrau.

${ }^{\circ-o}$ Legat von Bettzeug zugunsten eines minderjährigen Neffen.

p-p Verfügung eines Weingartens zugunsten desselben Neffen.

q-q Bei Eintritt in den Deutschen Orden Abtretung dieses Weingartens an dessen Vater, den Bruder des Erblassers.
} 
daz der vorgenant Niclas in Dewtschenherren orden farn wolt, so sol er desselben weingartens abtreten und mein pruder suellen sich dann desselben weingartens underwinden?. Item 'schaff im das pesst fudear wein, so es in meinem chellear leit, damit er denselben weingarten paw'. Und sdenselben knaben enphilch ich meinem lieben pruder Hannsen dem Schaffswol vor Werdertoer ze Wienn zu getrewr hannts und als ich im des vor meaniklich wol getraw. tBescheach aber, das derselb mein pruedear den benanten knaben zu getrewr hannd nicht innhield, so schaff und enphilch ich denselben chnaben Petrein dem Unger, dem floeczear, daz er in mit leib und mit guet innhald, als ich im auch des vor meanikleich wolgetraw. Und uschaff im ain chissten und amen graben seydl und ainem newn pelcz und ainen swarczen seydlu. Item darnach "schaff ich Agnesen, meins bruder tochtear, ain pet und zwaye leylachen und zwaye kuess. Und schaff ier auch ain was wein $z u$ sechs und dreyssig emmearn ${ }^{v}$. Item so ${ }^{w}$ schaff ich syben phund phenning hincz Sand Stephan ze Wienn $z u$ dem paw. Item auch schaff ich syben phund phenning hincz Sand Michel zu dem paw. Item so schaff ich hincz den Weissenbruedearn zu dem paw syben phund phenningw. Item darnach ${ }^{x}$ schaff ich meinem peichtuater hern Mertten dacz Sand Stephan zwaye

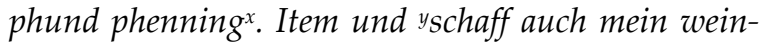
geartel auf der Herczogenpewent dem vorgenanten meinem bruder Hannsen dem Schaffswol, also daz er

\footnotetext{
r-r Weinlegat zugunsten des Bruders.

s-s Bestellung des Bruders zum Sachwalter des minderjährigen Neffen.

${ }^{\mathrm{t}-\mathrm{t}}$ Bestellung eines Ersatz-Sachwalters für den minderjährigen Neffen.

u-u Kleidungslegat zugunsten des Ersatzsachwalters.

${ }^{\mathrm{v}-\mathrm{v}}$ Wäsche- und Weinlegat zugunsten der Nichte des Erblassers.

w-w Stiftungen zum Kirchenbau zugunsten von St. Stephan und St. Michael sowie des Deutschen Ordens.

${ }^{x-x}$ Verfügung eines Geldlegats zugunsten des Beichtvaters des Erblassers.

y-y Verfügung eines Weingartens zugunsten des Bruders des Erblassers unter Auflage einer Jahrtagsstiftung.
}

mir von demselben weingarten ainen ewigen jartag ausrichten und begeen lassen sol mit vigilye und selambt und mit vier gesprochen messen darunder in Unser Frawn und Sand Johans capelln vor Werdertoer ze Wienn mit ainem halben phund geltes, alsuerr daz geraihen mag nach seinen trewn ${ }^{y}$. Item so ${ }^{z}$ schaff ich meinem brudear Ulreichen dem Schaffswol ze Swadorf meinen plaben seydl den langen. Item und schaff auch meinem bruder Hannsen dem Schaffswol ainen silbrein pechear und zehen phund beraitter phenningz. Item und ${ }^{a a}$ Schaff den chuerczen seydl den fuechseinn in das spital vor Kernertor ze Wienn, daz man den verchauf und dasselb gelt geb armen leweten in demselben spital von hannt ze hannt alsuerr daz geraichen mag und darczue ainen kanczen kern hauffen denselben armen lewten zu dem pad durch got und meiner sel hails willen ${ }^{a}$. Item darnach ${ }^{b b}$ schaff ich in dasselb spital zwelf phuend phenning und dasselb gelt sol man anlegen an ein erb, daz man den vorgenanten armen lewten alle iar dauon raihen und dienn sol ain phund phenning und daz geben von hannt ze hannt alsuerr das geraihen mag. Und sullen auch dieselben zwelf phund phenning angelegt werden an ein erb nach meiner brueder geuallen und mit ains yeden spitalmaister wissen. Wear aber das, daz dieselben mein bruedear [fol. 199v] also widerseczig wuerden, so sol und sich ain yeder spitalmaister desselben guets underwinden daran dieselben zwelf phund phenning gelegt worden sind ${ }^{b b}$. Item und ${ }^{c c}$ schaff Petreins des Ungers bruder kind zehen phund beraitter phenning. Item und schaff dem Prannttsteter funf phund phenning. Item und schaff dem Waschengiel auch funf phund phenning. Und schaff im meinen guten manntel den plaben. Und

\footnotetext{
z-z Sach- und Geldlegate zugunsten von Brüdern des Erblassers.

aa-aa Anordnung einer Seelheilstiftung zugunsten des Erblassers im Armenhaus vor dem Kärntnertor.

bb-bb Anordnung einer weiteren Armenstiftung im Armenhaus vor dem Kärntnertor im Einvernehmen zwischen den Brüdern des Erblassers und dem Spitalsmeister.

cc-cc Geld- und Sachlegate zugunsten familienfremder Personen.
} 
schaff im ain news schrot eysence. Item darnach ${ }^{d d}$ chaff ich zwayhundert mess, daz man mir die ausrichten und begeen sol durch got und meiner sel hails willen nach meiner geschefftherren rat, willen und wissen. Und schaff auch, daz man das vorgenant mein geschefft erst nach meinem tod also ausrichten

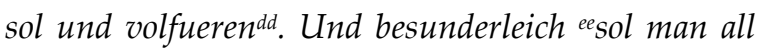
mein geltschuld von dem halben haus und von den stekchen und von vier fuedear weins und von anderm meinem holcz, daz ich dann hinder mein lass, ausrichten und beczaln. Und schaff, daz man dann von dem vorgenanten guet ain Ramfart ausrichten solee. Und ffwas dann der ubermass ist, das suellen die vorgenanten mein brudear miteinander tailn frewentleich an all mein geswistreid. Und schaff in auch all mein geltschuld, die man mir schuldig ist, wo sy darauf koemen muegen, den benanten meinen brudernff. Und ggacht guldein, die ligent in meiner taschen, die schaff ich, daz man mich damit erberleich bestatt und ainen stain auf mich darumb kaufgg. Das vorgenant hhmein gescheafft schaff und enphilch ich den erbern lewten, dem vorgenanten Hannsen dem Schaffswol, meinem lieben prudear, daz er das nach seinen trewen ausricht und volfuer, als ich das geordent und geschafft hab, als ich im des vor meanikleich wol getraw und als er got darumb antwurten sol am jungisten tagh. Und iibey dem vorgenanten meinem gescheafft sind gewesen die erbern lewt Meanndl der Vischer und Heinreich der Scheppach, baid burger ze Wienn, damit ich mein gescheafft also ausgericht und volfuert habii, als vorgeschriben und begriffen ist.

dd-dd Messstiftung zum Seelheil des Erblassers nach Anordnung der Willensvollstrecker.

ee-ee Anordnung über Tilgung von Schulden des Erblassers und Finanzierung einer Wallfahrt nach Rom "Ramfart").

ff-ff Teilung des von Verfügungen und Schulden frei gebliebenen Nachlasses („ubermass") sowie seiner Forderungen unter den Geschwistern des Erblassers. gg-gg Anordnung über Bestattung des Erblassers und Ausstattung seiner Grabstelle.

hh-hh Einsetzung eines Bruders des Erblassers als Willensvollstrecker.

ii-ii Nennung von zwei Stadtbürgern als Zeugen des Geschäfts.
ijAls darumb die obgenanten erbern lewt mit iern trewen an ayds stat vor unser in offem rat habent gesagt, als sy zurecht soltenij."

\section{Notariatsinstrument über ein Geschäft:}

Bestellung eines Willensvollstreckers; Verzeichnis der Schulden und Forderungen des Erblassers, Anordnung von Seelgeräten, Mess- und Jahrtagsstiftung; Verfügung zugunsten des Willensvollstreckers ${ }^{88}$

[Stadtbuch II, fol. 81r] ${ }^{89},{ }^{a}$ Anno domini CCCCmo nono. In nomine domini, amen. Anno natiuitatis eiusdem millesimo CCCCmo octauo die XVIma mensis decembris hora terciarum vel quasi indicione prima pontificis sanctissimi in Christo patris ac domini nostri domini Gregorii diuina prouidencia pape XIImi anno secundo ${ }^{b}$ in mei notarii publici testium subscriptorum presencia personaliter constitutus $^{b}$ chonorabilis vir dominus Nicolaus Johannis de Hunczing rector altarium Beate Marie Virginis et Undecim Milium Virginum in ecclesia Sancti Stephani ac Sancti Erasmi in ecclesia Sancti Michaelis Wienne Patauiensis diocesis per dei graciam sanus mente et racione licet langueus corpore $d_{\text {rerum }}$

ji-ji Eidesstattliche Erklärung der Zeugen.

88 JARITZ, NeschWARA, Stadtbücher 3, Nr. 1557; die dem edierten Text des Stadtbuches beigefügten textkritischen Anmerkungen von Gerhard Jaritz bleiben hier weg.

${ }^{89}$ Index: „Item instrumentum testamenti domini Nicolay de Hunczing".

a-a Invokation und mehrfache Datierung: Jahr, Monat, Tag und Stunde nach der Zeitrechnung (Inkarnationsjahr); Indiktion (,indictione prima“) berechnet sich wie folgt: $(1408+3): 15=94$, Rest 1 = die Indiktion; das Pontifikationsjahr ist 2 (Papst Gregor XII. wurde 1406 gewählt).

b-b Promulgatio (Publicatio): Erklärung des Notars über die Errichtung einer Notariatsurkunde.

${ }^{c-c}$ Namentliche Nennung des Testators, seiner Profession und Diözesanzugehörigkeit sowie Hinweis auf seine Geschäftsfähigkeit.

${ }^{\mathrm{d}-\mathrm{d}}$ Hervorhebung der zur angegebenen Zeit in deutscher Sprache getroffenen Verfügungen als letzter Wille des Erblassers, welcher in dieses Notariatsin- 
suarum ac bonorum omnium disposicionem per presentem testamentum ore proprio fecit et ordinauit prout in hoc presenti instrumento inferius de verbo ad verbum in thewtonico vulgariter continetur volens expresse et solempniter protestando ibidem, quod sit presens ordinacio ultime sue voluntatis. Valere debet tam iure testamenti quam et iure codicillorum vel alterius cuiuslibet ultime hominis voluntatis. Tenor vero eiusdem testamenti in thewtonico per ipsum dominum Nicolaum expressati ac totaliter conscripti per omnia sequitur in hec verbad. Von erst emphilich ich Niclas mein sel dem almechtigen got, der junckfrawn Marien und allen gots heiligen, wan ich schaiden sol von disem eillend. Darnach so emphilich ich meinem peichtuatter und landsman hern Hainreichen dem Weissen, kormaister zu Sand Stepfan hie ze Wienn, als mein gut und hab geistlich und weltlich, es sey gotsgab, weingerten, varunde und unvarunde hab, ynnerhalb und ausserhalb des lands ze Osterreich, wa die gelegen sein oder wer die inn hat und wie die genant sein oder wer die ynne habent, daz derselb kormaister innem und innhab und auch all mein geltschuld, die hernach begriffen ist von den, die mir gelten sullen, inbringen und mein gescheft alls ausricht und volbringe nach meiner selhaile. Item so $f_{\text {tun }}$ ich kunt, daz ich mit meinem wirt Jorgen dem Stewdlein, pinter hie ze Wienn, uberain komen pin und auch mich zu im in sein haus kost gedingt hab, also daz ich ein kamer in seinem haus gehabt han und mich in an seinen tischs und schussel geessen hab. Und bin in sein haus komen an Allerheiligen tag, da man zalt von Krists gepurdt vierczehenhundert jar und darnach in dem ersten jar, diezeit bin ich alle bei im gewesen, daz ich im alle jar,

strument wortwörtlich inseriert wird; Beifügung der sogenannten Kodizilliarklausel.

e-e Bestellung des Beichtvaters als Willensvollstrecker zur Abgeltung der Schulden des Erblassers sowie zur Durchführung seiner Anordnungen, insbesondere der Seelheilstiftungen.

${ }^{\mathrm{f}-\mathrm{f}}$ Hinweis auf offene Zahlungen des Erblassers an seinen Kost- und Quartiergeber und Aufrechnung mit seit 1401 vom Erblasser selbst oder dessen Schuldnern an diesen erbrachte Leistungen. alslang ich bey im wolt beleiben, scholt geben $12 \mathrm{tl}$. dn. fur ezzen und fur trencken und gemach. Er sol mir auch alle mal geben semel uber den tischs, so er sy gehaben mag. Daran hab ich demselben meinem wiert von erst beczalt und geben 13 guldein, die hat im von meinen wegen ingeantwurt maister Niclas von Furstenueld. Item so hat im von meinen wegen gegeben Iusti $28 \mathrm{tl}$. Item so hab ich ynn von erst bezalt, als seiner hausfrawn wol wissentlich ist, $12 \mathrm{tl}$. $d n$. von etlich kaufmanschaft, die er bestellt hett, und schuft sy bezalen hie ze Wienn nach dem und er sich gen Venedi erhub ze varen. Item darnach 3 tl. dn. Item $2 \mathrm{tl}$. an derselben kaufmanschaft. Item so hab ich im geben 24 emer weins. ain emmer umb ain halbphunt mynner $10 \mathrm{dn}$. Item so hab ich demselben meinem wiert geben 27 emer weins oder most, ye ain emmer umb $18 \mathrm{dn}$. phening. Item so hab ich im geben edels gestain fuer 2 flor. Item so ich geben demselben meinem wirt ein messpuch, das wol 24 tl. wert was, das hat er auch selb verkaufft und hat das gelt selb inn ${ }^{\mathrm{f}}$. Item gso beleibt mir Thomas der Englisch $12 \mathrm{tl}$. dn. an ainem weingarten, der zu Sand Erasm altar gehort dacz Sand Michel, den ich im hab gelassen mein lebtag alle jar umb $12 \mathrm{tl}$. dn., den er im zway jar hat gehabt und ain jar hat mich beczalt, das ander nicht. Auch ist er mir schuldig die zerung, die ich von seinen wegen hie vor dem official ze Wienn getan hab. Item so beleibt mir Anthonius der Arczt an dem Altenkolmarchk $4 \mathrm{tl}$. dn., die ich im gelichen hab zu der weich seins altars dacz Sand Michel. Item derselb Anthoni beleibt mir auch ain pfunt phenning von des krigs wegen, darumb im wol kunt ist. Item die Damin die Fleischhachkerin bey Sand Laurenczen uber sol mir gelten yeczund zu sand Michels tag von der mess wegen [fol. 80v] zu Sand Steffan $4 \mathrm{tl}$. dn. Item so sol mir gelten fraw Messrerin in der Kiemser haus bey den Predigern bey der Hochenschul yeczund zu der quatember ain pfunt phening. Item so sol mir gelten zu zins yeczund zu den quatembern die Zwetlerin bey Sand Merten $4 \mathrm{tl}$. dn. Item so sol mir gelten yeczund zu den quatembern Ulreich gesezzen in der

g-g Verzeichnis der offenen Forderungen des Erblassers. 
Alten Stenglin haus $80 \mathrm{dn}$. Item so sol man mir gelten 12 schilling phenning von dem haus Peters von Prabna in der Wolczeil yeczund zu den nechsten quatembern. Item so ist mir Hans Stichel, der kursner, von meiner weingarten wegen, die er ettlich jar hat gepaut, noch schuldig $18 \mathrm{tl}$. phening. Item so hab ich behabt zwo urtail wider Mathiam Iusti, aine vor dem abbt dacz den Schotten und aine vor dem official hie ze Wienn, damit ich behabt han wol hundert pfunt phenning. Item so beleibt mir Niclas der Weispacher zehen pfunt phening fur die mess, die ich Ulreichs des Zincken, dem got gnad, und seiner voruodern sel ze hilff und ze trost funfthalb jar all tag teglich zu Unser Frawn auf der Stetten ze Wienn gelesen hab an all sawmnuezz. hDie vorgenant geltschuld alle, die schaff ich zu meinem haus hie ze Wienn, daz die kapplen, die dann werdent daselbs nach meinem tod inbringen mitsambt hern Hainreichen dem Weissen, dem kormaister, meinem gescheftherren, daz die dasselb haus damit pawn und auch meiner sel nach iren trewn gedencken ${ }^{\mathrm{h}}$. Item iso tun ich ze wissen, daz ain frume andechtige erbere fraw mut het, alls ir guet, das sy hie hett ze Wienn, ze geben ze pessrung der egenanten meiner mess hie zu Stephan ze Wienn. Und darumb so verhies ich ir ze lassen ledichlich ir lebteg ain virtail in dem egenanten meinem haus. Wie die nu ist abgeweist worden, daz sy das nicht hat getan und an mir ir nichts abgegangen ist, so hab ich ir auch das virtail des egenanten meins hauss nicht lassen wellen wideruaren und han das geben und gab auch das dem egenanten Hainreichen dem Weissen, dem kormaister, meinem peichtuatter und meinem gescheftherren, daz der got fuer mich und all mein voruodern darumb pitt und daz er auch das paw, als im das fueg, also daz dasselb virtail nach seinem tod den vorgenanten meinen messen zu Sand

\footnotetext{
h-h Anweisung an den Willensvollstrecker, diese AuBenstände für den Bau einer Kapelle im Haus des Erblassers zu verwenden.

i-i Aufkündigung der Zusage eines Wohnrechts im Haus des Erblassers gegen die Verpflichtung zur Ergänzung der Messstiftung zugunsten des Erblassers.
}

Stephan beleibi. Item 'so schaff ich mein messpuch und ain klaine kisten in meiner kamer zu meinem altar zu Sand Micheli. Item ${ }^{k_{S O}}$ schaff ich und main, daz mein jartag und und auch der frawn jartag mit den 18 schilling phening gelts auf des egenanten Petrein von Prabna haus, darumb ain brief ist gegangen, werd jerlich, als derselb brief lautt, an alles vercziehen'. 'Super quibus omnibus et singulis prefatus dominus Nicolaus Johannis de Hunczing sibi per me notarium publicum infrascriptum unum vel plura publicum seu publica fieri petiit instrumental. ${ }^{m}$ Acta sunt hec Wienne dicte Patauiensis diocesis in domo Georii Stewdel prelibati opidani opidi Wiennensis in quadam kamera anno die mensis hora indicione et pontificis quibus supra presentibus honorabilibus et discretis viris dominis Larencio Friderici de Newnburga Klaustrali ortenario, Paulo dicto Gerstner de Noua Ciuitate vicario ecclesie Omnium Sanctorum alias Sancti Stephani ibidem Wienne et Fridrico dicto Fridolffinger de Ranshouen presbitero et clerico dicte Patauiensis diocesis et Salczburgensis diocesis et aliis pluribus fide dignis testibus ad premissa vocatis specialiter et rogatis et cetera ${ }^{m}$. ${ }^{n}$ Et ego Gundakerus Aschpechk de Obernperg clericus Patauiensis diocesis publicus apostolica et imperiali auctoritate notarius ${ }^{90}$ quia dicti testamenti ordi-

j-j Verfügung des Messbuches und anderer Sachen des Erblassers zugunsten des Willensvollstreckers.

k-k Anordnung einer Jahrtagsstiftung für den Erblasser und seine Frau.

${ }^{1-1}$ Hinweis auf den Beurkundungsauftrag (Rogation) des Erblassers an den Notar zur Errichtung einer Notariatsurkunde über den vorgetragenen Sachverhalt.

$\mathrm{m}-\mathrm{m}$ Angabe über den Ort der Errichtung der letztwilligen Verfügung und Anführung der anwesenden drei Zeugen.

n-n Publizitätswirksame Beurkundungsformel: Namentliche Nennung des Notars unter Hinweis auf seine Eigenschaft als öffentliche Beurkundungsperson kraft päpstlicher und kaiserlicher Autorisation; Erklärung des Notars, dass er die Notariatsurkunde selbst ausgefertigt habe; Hinweis auf seine eigenhändige Unterschrift und die Beifügung des Notariatssignets. ${ }^{90} \mathrm{Zu}$ Notar Gundaker Aschpechk von Obernberg: NESCHWARA, Notariat 161, 674, 684. 
nacionem et disposicionem omnibusque aliis et singulis premissis dum sic ut premittitur fieret et agerentur unacum prenominatis testibus presens interfui eaque sic fieri vidi et audiui. Ideoque hoc presens publicum instrumentum per alium me aliis ocupatis negociis fideliter scriptum exinde confeci ac publicam propria manu mea hic me subscribendo signoque et nomine meis solitis et consuetis signaui rogatus et requisitus in fidem et testimonium omnium premissorum ${ }^{n}$.

\section{Literatur:}

Paul BAUR, Testament und Bürgerschaft. Alltagsleben und Sachkultur im spätmittelalterlichen Konstanz (Sigmaringen 1989).

Wilhelm BRAUNEDER, Eine Übertragung Wiener Rechts auf Klosterneuburg („Aufsatz des Erbrechts 1381/83“), in: Wiener Geschichtsblätter 32 (1977) 236-237.

Wilhelm BRAUnEDER, Gerhard JARITZ (Hgg.), Die Wiener Stadtbücher 1395-1430, Teil 1: 1395-1400 (=Fontes Rerum Austriacarum, 3. Abt.: Fontes Iuris, Bd. 10,1, Wien-Köln 1989).

Wilhelm BRAUNEDER, Die Geltung obrigkeitlichen Privatrechts im spätmittelalterlichen Wien, in: DERS., Studien I: Entwicklung des öffentlichen Rechts (Frankfurt am Main u.a. 1994) 401-408.

Wilhelm BRAUNEDER, Typen des mittelalterlichen Erbrechts in ihrer Bedeutung für die Bevölkerungsentwicklung, in: DERS., Studien II: Entwicklung des Privatrechts (Frankfurt am Main u.a. 1994) 331-355

Wilhelm BRAUnEDER, Gerhard JARITZ, Christian NESCHWARA (Hgg.), Die Wiener Stadtbücher 13951430, Teil 2: 1401-1405 (= Fontes Rerum Austriacarum, 3. Abt.: Fontes Iuris, Bd. 10,2, Wien-Köln 1998).

Peter Csendes (Hg.), Die Rechtsquellen der Stadt Wien (= Fontes Rerum Austriacarum, 3. Abt.: Fontes Iuris, Bd. 9, Wien-Köln-Graz 1986).

Heinrich DeMELIUS, Zur Entstehung des Wiener Eisenbuches, in: Jahrbuch des Vereins für Geschichte der Stadt Wien 14 (1958) 47-50.

Christa DERNDARSKY, Analyse von Testamenten aus den Jahren 1395-1397 aus den Wiener Testamentsbüchern unter besonderer Berücksichtigung der vermachten Sachgüter. Ein Beitrag zur Alltagsgeschichte des ausgehenden 14. Jahrhunderts, (phil. Dipl.arb. Univ. Wien 2007).
Heinz HolzHAUER, Unterschrift, Unterfertigung von Urkunden, in: HRG ${ }^{1}$, Bd. 5 (Berlin 1998) 526-535.

Gerhard JARITZ, Christian Neschwara (Hgg.), Die Wiener Stadtbücher 1395-1430, Teil 3: 1406-1411, (=Fontes Rerum Austriacarum, 3. Abt.: Fontes Iuris, Bd. 10,3, Wien-Köln-Weimar 2006).

Gerhard JARITZ, Christian Neschwara (Hgg.), Die Wiener Stadtbücher 1395-1430, Teil 4: 1412-1417 (=Fontes Rerum Austriacarum, 3. Abt.: Fontes Iuris, Bd. 10,4, Wien-Köln-Weimar 2009).

Gerhard KÖBLER, Zielwörterbuch europäischer Rechtsgeschichte (Gießen ${ }^{52009) .}$

Wolfgang KLÖTZER, Stadtbuch, in: HRG ${ }^{1}$, Bd. 5 (Berlin 1998) 1849-1851.

Hans LENTZE, Der Rat und das Wiener Testamentsrecht des Mittelalters, in: Wiener Geschichtsblätter 2 (1947) 31-35.

Hans LENTZE, Begräbnis und Jahrtag, in: ZRG KA 36 (1950) 328-364.

Hans LENTZE, Die Rechtsform der Altarpfründen im mittelalterlichen Wien, in: ZRG KA 37 (1951) 221302.

Hans LeNTZE, Das Wiener Testamentsrecht des Mittelalters, 2 Teile, in: ZRG GA 69 (1952) 98-154 und 70 (1953) 129-229.

Hans LENTZE, Das Seelgerät im mittelalterlichen Wien, in: ZRG KA 44 (1958) 35-103.

Klaus LOHRMANN, Grundbücher (=Veröffentlichungen des Wiener Stadt- und Landesarchivs, Reihe A: Archivinventar, Serie 1 [Stadtarchiv], Heft 2, Wien 1986).

Hans-Dieter LoOsE, Hamburger Testamente 1351 bis 1400 (Hamburg 1970).

Hermine LUTZ, Alltagskultur und Lebensverhältnisse im Spiegel der Wiener Testamentsbücher (18. November 1395 bis 11. Dezember 1403) (phil. Diss., Univ. Wien 1983).

Uta MARQUARDT, „.... und hat sein Testament und letzten Willen also gemacht". Görlitzer Bürgertestamente des 16. Jahrhunderts (Leipzig 2009).

Judit Majorossy, Katalin Szende (Hgg.), Das Preßburger Protocollum Testamentorum 1410 (1427)1529, Teil 1: 1410-1487 (=Fontes Rerum Austriacarum, 3. Abt.: Fontes Iuris, Bd. 21,1, Wien-KölnWeimar 2010).

Wolfgang MAYER, Handschriften (= Veröffentlichungen des Wiener Stadt- und Landesarchivs, Reihe A: Archivinventar, Serie 3: Sammlungen, Heft 1, Wien 1986).

Christian NeschWARA, Geschichte des österreichischen Notariats, Bd. 1: Vom Spätmittelalter bis zum Erlass der Notariatsordnung 1850 (Wien 1996). 
Christian NESCHWARA, Rezensionen über:

Henning STEINFÜHRER (Hg.), Die Weimarer Stadtbücher des späten Mittelalters. Edition und Kommentar (=Veröffentlichungen der Historischen Kommission für Thüringen, Große Reihe 11, Köln-Weimar-Wien 2005);

Jenö HÁzi, Janos NemEth (Hgg.), Gerichtsbuch/Bírósági köny 1431-1531 (= Quellen zur Geschichte der Stadt Ödenburg, Reihe A, 2, Sopron 2005);

Károly MolLAY, Károly GoDA (Hgg.), Gedenkbuch/Feljegyzésy könyv 1492-1543 (= Quellen zur Geschichte der Stadt Ödenburg, Reihe A, 3, Sopron 2005)

alle in ZNR 33 (2011) (im Erscheinen).

Christian Neschwara, Grundbuch, in: EdN, Bd. 4 (Stuttgart-Weimar 2006) 1157-1159.

Werner OGRIS, Erbenwartrecht, in: HRG ${ }^{1}$, Bd. 1 (Berlin 1972) 958-959.

Werner OGRIS, Erbgut, in: HRG 1 , Bd. 1 (Berlin 1972) 963-964.

Werner OGRIS, Freiteil, in: HRG ${ }^{1}$, Bd. 1 (Berlin 1972) 1249-1251.

Werner OGRIS, Kaufgut, in: HRG', Bd. 2 (Berlin 1978) 666-667.

Werner OGRIS, Schenkung, in: HRG ${ }^{1}$, Bd. 4 (Berlin 1990) 1382-1384.

Werner OGRIS, Testament, in: $\mathrm{HRG}^{1}$, Bd. 5 (Berlin 1998) 152-166.

Werner OGRIS, Christian NESCHWARA, Erbenlaub, in: HRG $^{2}$, Bd. 1 (Berlin 2008) 1360-1361.

Werner OGRIS, Freiteil, in: HRG ${ }^{2}$, Bd. 2 (Berlin 2010) 1782-1784.

Ferdinand OPLL, Das große Wiener Stadtbuch, genannt „Eisenbuch“. Inhaltliche Erschließung (= Veröffentlichungen des Wiener Stadt- und Landesarchivs, Reihe A: Archivinventar, Serie 3: Sammlungen, Heft 4, Wien 1999).

Josef PAUSER, „in sterbenden leuffen, der wir dann teglich nach dem willen des allmechtigen gewartten muessen“. Das Seuchentestament in der
Wiener Stadtordnung von 1526, in: Gerald KOHL, Christian NeschwARA, Thomas SIMON (Hgg.), Rechtsgeschichte mit internationaler Perspektive. Festschrift zum 65. Geburtstag von Wilhelm Brauneder (Wien 2008) 477-498.

Uta REINHARDT, Lüneburger Testamente des Mittelalters 1323 bis 1500 (Hannover 1996).

Franz STAUB (Bearb.), Die ältesten Kaufbücher (13681388) (=Quellen zur Geschichte der Stadt Wien 3. Abt.: Grundbücher, Bd. 1, Wien 1898).

Franz STAUB (Bearb.), Gewerbuch B (1373-1419). Verbotbuch (1373-1399) (= Quellen zur Geschichte der Stadt Wien 3. Abt.: Grundbücher, Bd. 2, Wien 1911).

Franz STAUb (Bearb.), Das Satzbuch A (1373-1388) (Quellen zur Geschichte der Stadt Wien 3. Abt.: Grundbücher, Bd. 3, Wien 1911).

Karl WAHLE, Die Wiener "Genannten“ als Urkundspersonen, in: MIÖG 34 (1913), 636-652.

Friedrich WALTER, Die sogenannten Wiener Testamentenbücher (1395-1430) (Eine realienkundliche Untersuchung) (Prüfungsarbeit Institut für Österreichische Geschichtsforschung Wien 1923).

Dieter WERKMÜLLER, Rechtsgeschäfte auf den Todesfall in den deutschen Rechten des Spätmittelalters, in: Actes à cause de mort II: Europe médiévale et moderne (= Recueils de la Société Jean Bodin pour l'Histoire Comparative des Institutions 60, Bruxelles 1993) 257-266.

Gunter WESENER, „Einantwortung“ - Herkunft, Anwendung und Bedeutungen dieses Ausdrucks, in: Markus StePpan, Helmut GeBHARDT (Hgg.), Zur Geschichte des Rechts. Festschrift für Gernot Kocher zum 65. Geburtstag (Graz 2006) 485-494.

Gunter WESENER, Ephemere Besonderheiten des spätrömischen Erbrechts. Zur Frage des Fortlebens rechtlicher Institute, in: Holger ALTMEPPEN, Ingo REICHARD, Martin Josef SCHERMAIER (Hgg.), Festschrift für Rolf Knütel zum 70. Geburtstag (Heidelberg 2009) 1401-1422. 